\title{
Percutaneous computed tomography- guided radiofrequency thermal ablation of small unresectable lung tumours
}

\author{
S. Rossi*, R. Dore" , A. Cascina", V. Vespro\#, F. Garbagnati', L. Rosa*, V. Ravetta*, \\ A. Azzaretti ${ }^{\#}$, P. Di Tolla ${ }^{+}$, G. Orlandoni ${ }^{\S}$ and E. Pozzi ${ }^{\oplus}$
}

ABSTRACT: The aim of the current study was to evaluate the safety and the efficacy of radiofrequency thermal ablation (RFTA) for the treatment of nonsmall cell lung cancer (NSCLC) and isolated pulmonary metastases (METs) from colorectal cancer (CRC).

A total of 31 patients (15 with NSCLCs and 16 with CRC lung METs), with 36 lung tumour nodules (mean \pm SD diameter: $22 \pm 8 \mathrm{~mm}$, range: $10-35 \mathrm{~mm}$ ) underwent computed tomography (CT)-guided RFTA using expandable electrodes. Contrast-enhanced CT was performed before and after (immediately and $30 \pm 5$ days) each RFTA session to assess immediate results and complications and repeated 3 and 6 months post-RFTA, as well as every 6 months thereafter, to evaluate long-term results. Complete radiological necrosis was defined as a nonenhancing area at the tumour site that was equal to or larger than the treated tumour; persistence of enhancement at the tumour site indicated incomplete treatment. Local recurrence was defined as an increase in tumour size and/or enhancing tissue at the tumour site.

Complete radiological necrosis of the 36 tumours was achieved with 39 RFTA sessions and 42 electrode insertions. No major complications or deaths were observed. Six patients experienced mild-to-moderate pain during the procedure. There were five cases of pneumothorax, none requiring drainage and four cases of pneumonia, which were successfully treated with antibiotics. After a mean follow-up of 11.4 \pm 7.7 months (range of 3-36 months), the overall local recurrence rate was $13.9 \%$ ( 20 and $9.5 \%$ for NSCLC and CRC-METs patients, repectively). Nineteen of the 31 (61.3\%) patients were alive (15 apparently disease free) and 12 (38.7\%) had died (three from causes unrelated to their cancer).

Radiofrequency thermal ablation seems to be a safe, effective method for producing complete ablation of small nonsmall cell lung cancers and pulmonary colorectal cancer metastases.

KEYWORDS: Lung neoplasms, lung tumours, radiofrequency thermal ablation

urgical resection is the treatment of choice for primary nonsmall cell lung cancers (NSCLCs) and isolated pulmonary metastases (METs) from colorectal cancer (CRC) [1,2]. However, approximately two-thirds of all NSCLC patients are ineligible for curative resection due to tobacco-related comorbidity, concomitant extrapulmonary diseases and/or advanced age $[3,4]$. Surgery is excluded in a similar percentage of pulmonary CRC-METs patients due to the presence of multifocal disease [5]. In any case, the surgical approach is by no means free from complications, including mortality, and it is difficult to repeat for recurrences $[6,7]$. Systemic chemotherapy and radiation therapy are often the only options offered to lung cancer patients, but these approaches produce substantial increases in survival only in small subsets of highly selected cases $[8,9]$.

Radiofrequency (RF) thermal ablation (RFTA) has proved to be effective in the treatment of liver tumours [10-12] and it has recently been evaluated by some groups as a minimally invasive therapeutic approach for malignant lung tumours [13-17]. The technique involves the delivery of RF energy through an electrode inserted in the tumour mass. Frictional heat is generated in tissues surrounding the conductive tip of the electrode where the density of the electric current is maximal [18]. This heat propagates through adjacent tissues and its distribution at any given instant can be described in terms of isothermal surfaces, whose values decrease as the distance from the electrode tip

\section{AFFILIATIONS}

${ }^{*} \mathrm{VI}$ Dept of Internal Medicine and Interventional Ultrasonography, \# Institute of Radiology, and

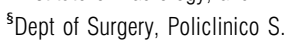
Matteo, I.R.C.C.S.,

"Dept of Respiratory Diseases, University of Pavia, Pavia, and +Dept of Radiology, National Cancer Institute, Milan, Italy.

CORRESPONDENCE

E. Pozzi

Dept of Respiratory Diseases

University of Pavia

I.R.C.C.S. San Matteo

P.le Golgi no 1

27100

Pavia

Italy

Fax: 393486717811

E-mail: ernesto.pozzi@unipv.it

Received:

May 032005

Accepted after revision:

November 282005

European Respiratory Journal

Print ISSN 0903-1936

Online ISSN 1399-3003 
increases. Tissue lying between the electrode tip and the $50^{\circ} \mathrm{C}$ isotherm undergo coagulative necrosis, the so-called thermal lesions, within $\sim 2 \mathrm{~min}$ [18]. In liver tissue, commercially available RF electrodes produce thermal lesions with diameters ranging 2.4-3.5 cm [10-12], which means that tumours $<3.5 \mathrm{~cm}$ in diameter can theoretically be ablated with a single electrode insertion.

RFTA of lung tumours has been performed without complications in rabbits [19]. In humans, it has been used to debulk endoluminal tumours of the bronchi [20] and to treat inoperable lung cancers [13-17], but the results are difficult to interpret. In each case, the group of patients treated was fairly small and by no means homogeneous, and this, together with differences in the RFTA protocols used in the various studies, might explain some of the contradictory results that have emerged. Furthermore, the follow-up was generally limited to the first months after treatment so the question of long-term efficacy also remains to be examined.

In the present study, the efficacy of RFTA for local control of small NSCLCs or pulmonary CRC-METs and the complications related to the procedure itself were evaluated.

\section{MATERIALS AND METHODS Patients}

The study protocol, which was fully concordant with the principles of the Helsinki Declaration, was approved by the Institutional Ethics Committee (Policinico San Matteo, I.R.C.C.S., Pavia, Italy). Each patient provided written informed consent to all study procedures after their nature had been carefully explained.

Between January 2002 and December 2004, 31 patients with NSCLCs or CRC-METs were enrolled in the study and scheduled for percutaneous RFTA. All 31 patients had been evaluated jointly by the anaesthesiology and surgical staff of Policinico San Matteo, I.R.C.C.S. In all cases, the patients' tumours were considered potentially resectable from an anatomic point of view, but surgical treatment was excluded due to unacceptably high surgical risk, as estimated by the anaesthesiologists, based on widely accepted criteria $[4,6]$ and/or patient refusal (table 1). All enrolled patients were managed jointly by Policinico San Matteo, I.R.C.C.S. medical and oncology staff.

The enrolment criteria listed in table 1 were verified by means of laboratory, radiological and endoscopical examinations. Intrathoracic disease extension was assessed by full-chest spiral computed tomography (CT; Sensation 16; Siemens, Erlangen, Germany). Scans were obtained prior to and $90 \mathrm{~s}$ after power injection of $100-120 \mathrm{~mL}$ contrast medium (Iomeron; Bracco, Milan, Italy) at a rate of $2 \mathrm{~mL} \cdot \mathrm{s}^{-1}$. The enhancement characteristics of the tumour, its vertical, sagittal and traverse diameters, as well as its location were evaluated in multiplanar images (1-mm scan collimation; 5-mm-thick sections; $120 \mathrm{Kvp} ; 180 \mathrm{mAs})$. CT images were visualised using soft-tissue (300 W/50 M) and lung (1700 W/-700 M) windows. Tumour diagnosis was confirmed in all cases by CT-guided aspiration biopsy performed with a 22-gauge needle (Ecoject; HS Hospital Service, Cavezzo, Italy). Samples were processed by standard cytology and immunohistochemical staining methods [21]. Tumour-staging studies included routine

\begin{tabular}{lc}
\hline TABLE 1 Study enrolment criteria \\
\hline Inclusion criteria & Exclusion criteria \\
\hline $\begin{array}{l}\text { Type of lung tumour } \\
\text { NSCLC }\end{array}$ Tumour infiltration of the mainstem \\
bronchi, trachea and/or \\
mediastinum \\
Pathological diagnosis of tumour & NSCLC patients \\
& Stage II-IV \\
Maximum tumour diameter $\leqslant 3.5 \mathrm{~cm}$ & Extrapulmonary spread \\
& CRC lung METs patients \\
Number of tumour nodules $\leqslant 2$ & Platelet count $\leqslant 50,000 \mathrm{~mm}^{3}$ \\
& and/or prothrombin time $\leqslant 50 \%$ \\
Tumour surrounded by aerated & Concomitant disease with life \\
parenchyma & expectancy $<6$ months \\
Exclusion of surgery as a & Refusal of RFTA \\
treatment option &
\end{tabular}

NSCLC: nonsmall cell lung cancer; CRC-METs: pulmonary metastases isolated from colorectal cancer; RFTA: radiofrequency thermal ablation. ${ }^{*}$ : Exclusion of the surgical option was based on surgical risk, which was evaluated by members of the anesthesiology staff using widely accepted criteria $[4,6]$, or on the patient's refusal to undergo surgery.

haematology, renal and liver-function tests, stool assays for occult blood, and sonographical and spiral CT studies of the abdomen. All NSCLC patients also had bronchoscopy, spiral CT of the brain and whole body bone scintigraphy. The CRCMETs patients also underwent a colonoscopy. Other studies, e.g. body bone scintigraphy, bone-specific radiographs, organspecific spiral CT, biopsy, etc., were also performed when needed, based on symptoms and imaging findings. Pulmonary function was evaluated by spirometry and blood-gas analysis. For patients with NSCLCs, pulmonary function was classified according to the Global Initiative for Chronic Obstructive Lung Disease (GOLD) guidelines [22] and tumours were staged using the international system [23].

\section{Equipment and procedure}

The RF system consisted of a generator, an active electrode and grounding pads. The RF generators had a frequency of $480 \mathrm{kHz}$ and a power output of 100-200 W. Digital displays on the front of the device indicated the power output, impedance value and procedure time (Models RF $3000^{\mathrm{TM}}$; Boston Scientific Corporation, Natlick, MA, USA and TAG $100 \mathrm{~W}$; Invatec s.r.l., Concesio, Italy) [24]. The active electrodes used were capable of creating thermal lesions measuring 3.0$3.5 \mathrm{~cm}$ in diameter (fig. 1) [11]. The grounding pads were conductive plates measuring $8.0 \times 16.5 \mathrm{~cm}$ (Boston Scientific Corporation; and Hospital's Plate; GPS S.r.l., Mozzo, Italy).

The RFTA procedure was performed after an overnight fast, without a general anaesthetic or conscious sedation. Intravenous antibiotic coverage $(1 \mathrm{~g}$ cefotaxime t.i.d.) was started $3 \mathrm{~h}$ prior to the procedure and continued through the third post-operative day. Two to four grounding pads were attached to the patient's back and connected to the RF generator. The patient was then prepped in the prone, lateral, or supine decubitus position, depending on the preselected electrode 

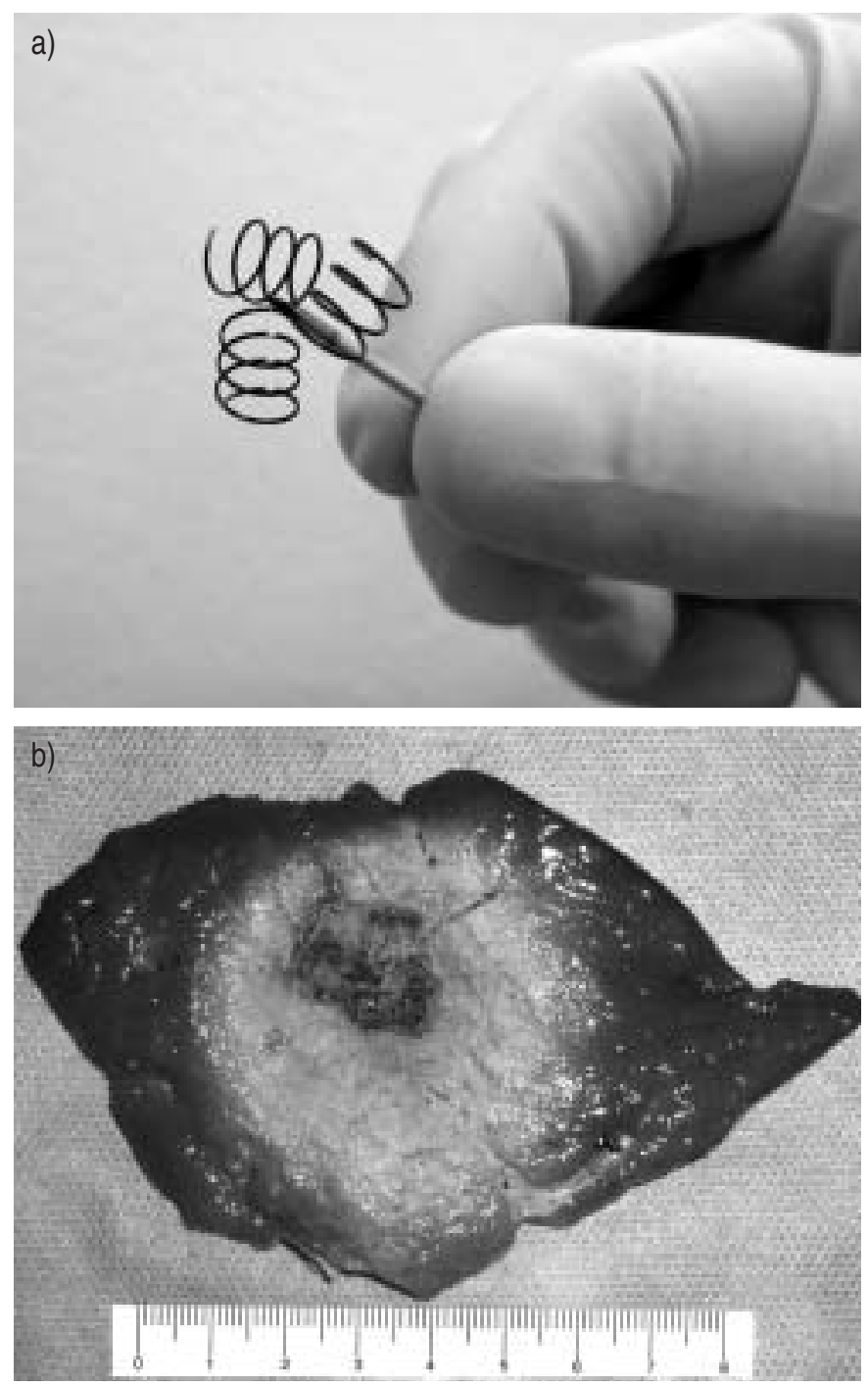

FIGURE 1. a) An electrode devise, used to perform radiofrequency thermal ablation. The electrode consists of an $18 \mathrm{G}$ stainless-steel 20-cm needle whose shaft is insulated with a 0.1 -mm-thick layer of plastic. The exposed tip, which is $1.0 \mathrm{~mm}$ long, contains three spiral arrays that, when deployed, extend $2.0 \mathrm{~cm}$ beyond the tip. They are deployed and retracted by means of a graduated control device on the electrode handle. b) Thermal lesion measuring $3.3 \times 3.5 \mathrm{~cm}$ in diameter produced in vivo in the liver of a pig, using an electrode similar to the one shown in a).

insertion tract (a straight line passing from the skin surface through an intercostal space to the centre of the tumour). The pathway chosen represented the shortest and most direct path to the tumour that did not intersect any major airways or blood vessels (i.e. those visible on the CT). Under CT guidance, a 22gauge Chiba (Ecoject, HS Hospital Service, Cavezzo, Italy) needle was passed through the chest wall and advanced towards the tumour along the pathway that would be used for the electrode insertion. As the needle advanced, $1 \%$ xylocaine (5-10 mL) was injected to anaesthetise the electrode tract, and when the needle tip reached the parietal subpleural space, an additional bolus of $10 \mathrm{~mL}$ was injected, as shown in figure 2 . This final injection not only anaesthetised the parietal pleura, it also compressed the underlying lung parenchyma, thus reducing the distance that the electrode would have to travel to reach the tumour. The type of active electrode used was selected based on the size and location of the tumour, and insertion was carried out under CT guidance. The tip was positioned in or near the centre of the tumour so that deployed arrays reached the farthest margin of the tumour and placement was verified by three-dimensional CT reconstruction (fig. 2b). The RF generator was started and power was progressively increased until impedance reached values that blocked further energy delivery [11, 24]. Depending on the shape and dimensions of the tumour, one or more thermal lesions were created along the electrode insertion tract using a pullback technique $[10,25]$. During each procedure, the power delivered, procedure duration and impedance values were recorded. Throughout the procedure, heart rate, blood pressure, ECG and blood oxygen levels were continuously monitored in a noninvasive manner, and the pain reported by the patient was scored as absent, mild discomfort (requiring no treatment), mild-to-moderate pain (requiring analgesics) or severe pain (necessitating interruption of the procedure).

\section{Immediate post-treatment and follow-up studies}

Spiral CT was performed at the end of the RFTA procedure for gross assessment of results and the detection of possible complications. The true assessment of the completeness of treatment was based on findings from a second spiral CT repeated $30 \pm 5$ days later. At this time, the hyperaemia, oedema and inflammatory tissue reaction provoked by the procedure itself, which can produce enhancement on posttreatment CT, are no longer present [10-12]. The presence of enhancing tissue on the $30 \pm 5$-day CT scan was thus regarded as indicative of incomplete treatment, whereas the presence at the tumour site of a nonenhancing area larger than the treated one was considered radiological evidence of complete tumour necrosis and, therefore, of successful treatment (fig. 2). In the former case, RFTA was repeated, as described previously, $\sim 1$ week after the residual tumour was detected. If enhancing tissue at the tumour site was observed $30 \pm 5$ days after the second RFTA session, the case was defined as a treatment failure. The patients whose $30 \pm 5$ day CT scan findings were suggestive of complete tumour necrosis underwent clinicalradiological follow-up that included spiral CT scans 3, 6 and 12 months after RFTA for the first year and every 6 months thereafter.

Mortality rates were calculated, as well as the frequencies of local recurrence (reappearance of enhancing tissue at the original tumour site), intrapulmonary disease progression (appearance of new lung tumours at sites other than the treated one), and extrapulmonary spread (NSCLC)/active extrapulmonary disease (CRC-METs).

\section{RESULTS}

Patient and tumour characteristics are summarised in table 2. Each of the 15 NSCLC patients had a single tumour and two of them had undergone lobectomies for NSCLCs of the contralateral lung. Eleven of the $16 \mathrm{CRC}$-METs patients had a single tumour, whilst the other five patients had two tumours. All of the metastatic tumour nodules had been detected or had presented signs of enlargement during a previous course of chemotherapy. 

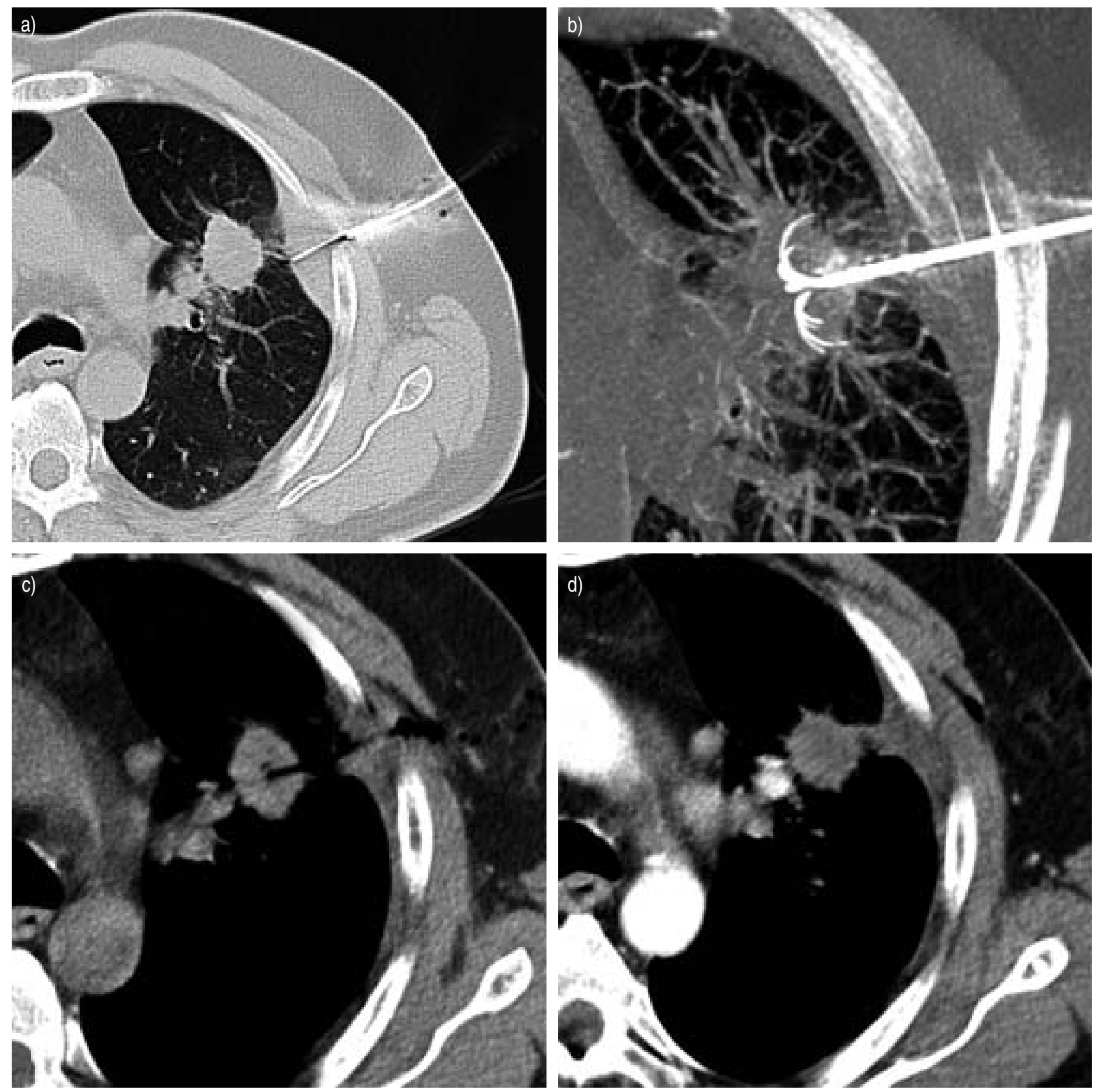

FIGURE 2. a) Computed tomography (CT) scan of the left lung of a colorectal cancer-pulmonary metastases (CRC-METs) patient. The metastatic nodule appears as a hyperdense mass $2.0 \mathrm{~cm}$ in diameter in the upper lobe (CT window 1700 W/-700 M). b) Three-dimensional CT reconstruction confirms the correct position of the electrode tip and its deployed arrays within the tumour mass (CT window 1700 W/- 700 M). c) Post-treatment CT densitometric evaluation of the tumour mass before injection of contrast medium (CT window $300 \mathrm{~W} / 50 \mathrm{M}$ ). d) Post-treatment CT densitometric evaluation of the tumour mass after injection of contrast medium demonstrated a lack of enhancement at the tumour site (CT window $300 \mathrm{~W} / 50 \mathrm{M}$ ).

Each of the 36 lung tumours was treated in a single RFTA session. In 33 cases, a single electrode insertion was sufficient; two insertions were required in three cases. A total of 22 tumours were treated with umbrella-tip electrodes and spiraltip electrodes were used for the remaining 14 tumours. The mean (range) duration time for the procedure was 19.5 (12-36) $\mathrm{min}$. The power delivered for each thermal lesion ranged from $30-100 \mathrm{~W}$ and the maximum impedance (i.e. the value measured immediately before the abrupt rise that interrupted further delivery) ranged from 68-143 Ohms (mean \pm SD $101 \pm 9.5 \mathrm{Ohms}$ ). None of the patients experienced severe pain. In 30 sessions, the procedure was pain-free or associated with slight discomfort. In the remaining six, mild-to-moderate pain was easily controlled with a single intravenous $100 \mathrm{mg}$ injection of tramadol (Contramal ${ }^{\mathrm{TM}}$; Grunental $\mathrm{GmbH}$, Stolberg, Germany). 


\begin{tabular}{|c|c|}
\hline Characteristics & \\
\hline Patients n & 31 \\
\hline \multicolumn{2}{|l|}{ Sex } \\
\hline Male & $28(90)$ \\
\hline Female & $3(10)$ \\
\hline Mean age \pm SD (range) yrs & $66.2 \pm 7.9(50-80)$ \\
\hline \multicolumn{2}{|l|}{ Karnofski score } \\
\hline $80-100 \%$ & $30(96.8)$ \\
\hline$<80 \%$ & $1(3.2)$ \\
\hline \multicolumn{2}{|l|}{ Tumour histology } \\
\hline Primary & $15(48.4)$ \\
\hline Adenocarcinoma/bronchioloalveolar & $12(80)$ \\
\hline Squamous-cell & $3(20)$ \\
\hline CRC-METs & $16(51.6)$ \\
\hline \multicolumn{2}{|l|}{ Tumour number } \\
\hline Single nodule & $26(83.9)$ \\
\hline Two nodules & $5(16.1)$ \\
\hline \multicolumn{2}{|l|}{ Mean tumour diameter ${ }^{\#} \mathbf{m m}$} \\
\hline$\geqslant 10-\leqslant 20$ & $19(52.8)$ \\
\hline$>20-\leqslant 30$ & $11(30.6)$ \\
\hline$>30-\leqslant 35$ & $6(16.6)$ \\
\hline \multicolumn{2}{|l|}{ Tumour location } \\
\hline \multicolumn{2}{|l|}{ Right lobe } \\
\hline Upper & $7(19.4)$ \\
\hline Middle & $1(2.8)$ \\
\hline Lower & $8(22.2)$ \\
\hline \multicolumn{2}{|l|}{ Left lobe } \\
\hline Upper & $10(27.8)$ \\
\hline Lower & $10(27.8)$ \\
\hline NSCLC patients $n$ & 15 \\
\hline \multicolumn{2}{|l|}{ GOLD } \\
\hline Class I & $9(60.0)$ \\
\hline Class II & $4(26.7)$ \\
\hline Class III & $2(13.3)$ \\
\hline \multicolumn{2}{|l|}{ Stage } \\
\hline $\mathrm{IA}$ & $13(86.6)$ \\
\hline IB & $2(13.4)$ \\
\hline
\end{tabular}

Data are presented as $\mathrm{n}$ (\%) unless otherwise stated. CRC-METs: pulmonary metastases isolated from colorectal cancer; NSCLC: nonsmall cell lung cancer; GOLD: Global Initiative for Chronic Obstructive Pulmonary Disease. \#: mean \pm SD (range) diameter was $22 \pm 8(10-35) \mathrm{mm}$

The results of the post-treatment and $30 \pm 5$-day follow-up CT scans are summarised in table 3 and figure 2 . The three patients whose post-treatment scans revealed incomplete radiological necrosis underwent a second RFTA session with one electrode insertion and the post-treatment CT scans revealed no residual enhancement. The treatment-failure rate for the original tumours was $0 \%$. A total of 39 RFTA sessions with 42 electrode insertions were required to treat 36 tumours.

There were no major complications or deaths. Post-treatment CT revealed pneumothorax in five cases $(11.9 \%)$, but all were self-limiting and did not require drainage. Other minor complications included: two cases $(4.7 \%)$ of mild transient haemoptysis; four cases $(9.5 \%)$ of pneumonia, which were

\begin{tabular}{|c|c|c|c|c|}
\hline \multirow[t]{3}{*}{ TABLE 3} & \multicolumn{4}{|c|}{$\begin{array}{l}\text { Post-treatment and } 30 \pm 5 \text {-day follow-up } \\
\text { computed tomography }(\mathrm{CT}) \text { findings for the } 36 \\
\text { lung tumours treated with radiofrequency } \\
\text { thermal ablation }\end{array}$} \\
\hline & & \multicolumn{3}{|c|}{ Tumour size mm } \\
\hline & & $\geqslant 10-\leqslant 20$ & $21-\leqslant 30$ & $31 \leqslant 35$ \\
\hline Patients n & & 19 & 11 & 6 \\
\hline \multicolumn{5}{|c|}{$\begin{array}{l}\text { CT scan immediately after } \\
\text { procedure }\end{array}$} \\
\hline Ground-9 & ass halo $\#$ & $19(100)$ & $11(100)$ & $3(50)$ \\
\hline \multicolumn{5}{|c|}{ Enhanced } \\
\hline Nonenha & cing area & $19(100)$ & $11(100)$ & $3(50)$ \\
\hline Residual & nhancing tissue & 0 & 0 & $3(50)$ \\
\hline $\begin{array}{r}\text { CT scan } 30 \pm \\
\text { procedure } \\
\text { Unenhance }\end{array}$ & -days after & & & \\
\hline Ground-g & ass halo & 0 & 0 & 0 \\
\hline \multicolumn{5}{|c|}{ Enhanced } \\
\hline Nonenha & cing area & $19(100)$ & $11(100)$ & $3(50)$ \\
\hline Residual & nhancing tissue & 0 & 0 & $3(50)$ \\
\hline
\end{tabular}

successfully treated by prolonging antibiotic therapy for 8 days; one case $(2.3 \%)$ of pleurisy, which was controlled by a $100-\mathrm{mg}$ oral administration of tramadol (Contramal ${ }^{\mathrm{TM}}$ ) t.i.d; and four cases $(9.5 \%)$ of isolated low-grade fever lasting 2-3 days.

In a mean follow-up of $11.4 \pm 7.7$ months (range: $3-36$ months), no long-term complications or evidence of tumour-cell seeding were observed. All 36 treated nodules were evaluated with spiral CT 3 months after RFTA. The vast majority (35 tumours, $97.2 \%$ ) appeared as nonenhancing areas that were $30-40 \%$ smaller than the original tumours; the remaining nodule $(2.8 \%)$ had increased in volume and presented clear enhancement. Twenty-four of the 36 nodules $(66.7 \%)$ were re-examined 6 months after RFTA. In 22 out of the $24(91.7 \%)$ cases, CT revealed nonenhancing areas $50-60 \%$ smaller than the original tumour; the remaining two $(8.3 \%)$ presented volume increases and enhancement. Almost half of the nodules $(16,44.4 \%)$ were re-examined at 12 months. Ten of these $16(62.5 \%)$ presented persistent nonenhancement and substantial shrinkage $(<60$ $80 \%$ of the original volumes) and four of these (25\%) were undetectable. The remaining two $(12.5 \%)$ nodules showed volume increases and enhancement. Of the nine nodules $(25.0 \%)$ examined at the 24-month follow-up, five $(55.6 \%)$ were still nonenhanced and $\sim 80 \%$ were smaller than the original tumour; in the remaining four cases (44.4\%), the only finding was a fibrotic scar at the site of the treated tumour nodule (fig. 3). As shown in table 4, the overall local recurrence rate was $13.9 \%$, while higher rates were recorded for intrapulmonary disease progression and extra lungspread/active extrapulmonary disease.

The six new lung metastases in CRC-METs patients were also treated with RFTA. Treatment was successful in five out of six 

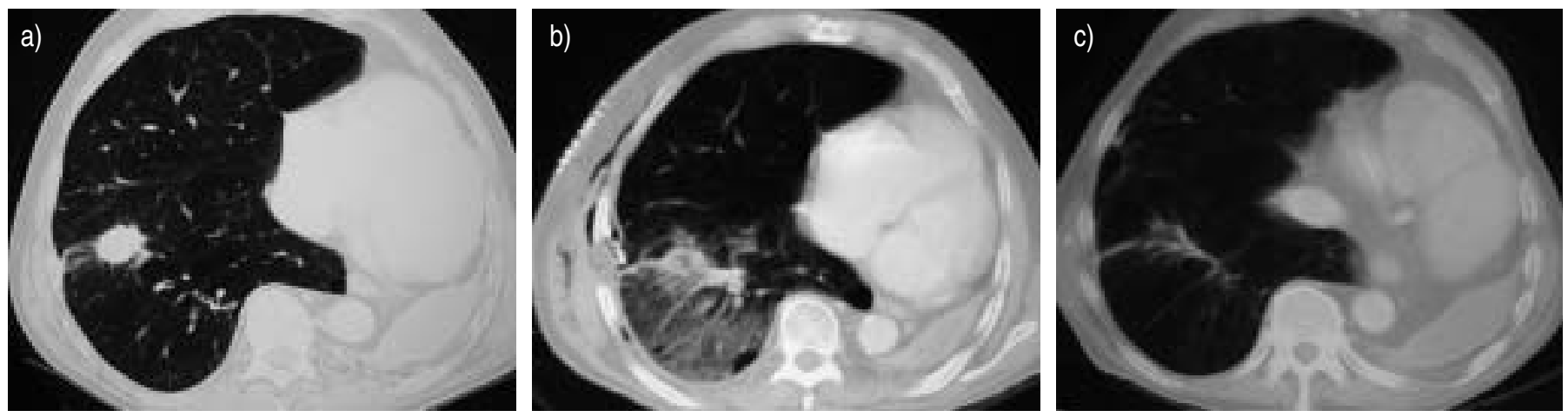

FIGURE 3. a) Computed tomography (CT) scans showed (CT window $1700 \mathrm{~W} /-700 \mathrm{M}$ ) a hyperdense mass $3.0 \mathrm{~cm}$ in diameter, representing a right lower lobe nonsmall cell lung cancer (NSCLC) in a patient who had previously had a left lobectomy for contralateral tumour. b) After radiofrequency thermal ablation, the tumour mass is surrounded by a ground-glass halo. c) The tumour mass is no longer visible 3-yrs later, and a thin hyperdense fibrotic scar is present at the site of the tumour

TABLE 4 Current status of treated patients ${ }^{\#}$

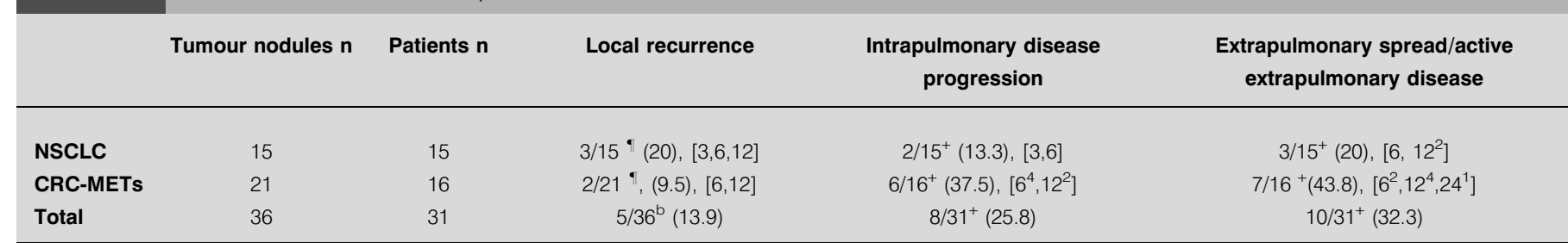

Data are presented as $n(\%)\left[x^{a}\right]$ where $x$ is the time of detection expressed in months after treatment and a refers to the number of patients if $>1$, unless otherwise stated. Local recurrences were calculated for each tumour nodule while intrapulmonary disease progression and extrapulmonary spread/active extrapulmonary disease were calculated for each patient. NSCLC: nonsmall cell lung cancer; CRC-METs: pulmonary metastases isolated from colorectal cancer. ${ }^{\#}$ : mean \pm SD (range) follow-up was $11.4 \pm 7.7$ (3-36) months; ${ }^{\circ}$ : treated tumours; ${ }^{+}$: treated patients.

cases; the remaining case was classified as a treatment failure. None of the procedures was associated with complications. Therefore, in the entire study, a total of 42 lung tumours were treated with RFTA, and in 41 out of $42(97.6 \%)$ the posttreatment $\mathrm{CT}$ findings revealed complete radiological ablation.

Case outcomes and causes of death are summarised in table 5 .

\section{DISCUSSION}

The results of the current study indicate that, when surgery is not an option, RFTA may be a safe and effective alternative for local control of NSCLC and pulmonary CRC-METs nodules $<3.5 \mathrm{~cm}$ in diameter. Follow-up spiral CT findings provided reliable evidence of complete tumour necrosis in $>80 \%$ of the nodules followed for $>12$ months. At the site of these nodules, CT currently reveals only fibrotic scars or hyperdense nonenhanced areas $60-80 \%$ smaller than the original tumours.

The major problem encountered with this approach is the need to confirm the completeness of the ablation. With all currently available radiological techniques, residual viable cancer tissue may be missed as a result of its limited volume or

\section{TABLE 5 Outcome of the treated patients ${ }^{\#}$}

\begin{tabular}{|c|c|c|c|c|c|c|c|}
\hline & Patients n & $\begin{array}{l}\text { Apparently } \\
\text { disease free }\end{array}$ & With disease & Total & $\begin{array}{l}\text { Apparently } \\
\text { disease free }\end{array}$ & $\begin{array}{l}\text { With disease } \\
\text { progression }\end{array}$ & Total \\
\hline NSCLC & 15 & $9(60)$ & $2(13.3)^{\pi}$ & $11(73)$ & $2(13.3)$ & $2(13.3)^{+}$ & $4(26.6)$ \\
\hline CRC-METS & 16 & $6(37.5)$ & $2(12.5)^{\S}$ & $8(50)$ & $1(6.3)$ & $7(43.7)^{f}$ & $8(50)$ \\
\hline
\end{tabular}

Data are presented as $n$ or $n(\%)$. NSCLC: nonsmall cell lung cancer; CRC-METs: pulmonary metastases isolated from rectal cancer. ${ }^{\#}:$ mean \pm SD (range) follow-up of $11.4 \pm 7.7$ (3-36) months; " : local recurrence and extrapulmonary spreads (metastases to the liver; $n=1$ ), intrapulmonary disease progression and extrapulmonary spreads (lymph nodes metastases; $n=1) ;{ }^{+}$: local recurrence and extrapulmonary spreads (brain metastases) $(n=1)$, local recurrence and intrapulmonary disease progression with pleural involvement $(n=1) ;$ : local recurrence and active extrapulmonary disease $(n=1)$, active extrapulmonary disease $(n=1) ;:$ intrapulmonary disease progression $(n=2)$, local recurrence and active extrapulmonary disease $(n=1)$, active extrapulmonary disease $(n=4)$ 
contrast-enhancement behaviour resembling that of necrotic tissue. Therefore, the absence of all enhancement at the tumour site is not an absolute indicator of complete tumour necrosis. CT-guided biopsy is of little help in these cases; even multiple biopsies provide data only on a limited volume of the treated tumour and results are conclusive only if they are positive. With all types of percutaneous tumour ablation procedures, adequate imaging follow-up is the only way to definitively exclude the presence of residual viable neoplastic tissue. Since recurrence caused by small foci of residual tumour can become evident up to 2 yrs after treatment [10], the success rates reported in the current study, which are based on a mean follow-up of $11.4 \pm 7.7$ months, may be slightly overestimated.

Nonetheless, spiral CT appears to be the most satisfactory technique for evaluating the immediate results and possible complications of RFTA. In previous studies of RFTA for neoplasms of the liver or lung [11-19], tumour necrosis has been considered to be complete when $\mathrm{CT}$ reveals a nonenhancing area at the tumour site, which is initially larger than the treated tumour. Indeed, when this finding was noted in the current patients' $30 \pm 5$-day CT scan, $>80 \%$ of the treated tumours drastically decreased in size during subsequent follow-up visits and eventually disappeared. On the immediate post-treatment CT scan, the hyperdense area representing the necrotic tumour area was generally surrounded by a ground-glass halo, which seems to correspond to a thin layer of heat-damaged normal lung parenchyma. This halo was observed in all cases in which no enhancing tissue was noted at the treated tumour site, but not in the three cases of incomplete treatment or in the single case of treatment failure. This may, therefore, be another important sign of satisfactory ablation. As in the liver, the coagulative necrosis seems to be confined to the tumour tissue and a very thin margin of normal lung parenchyma [10, 26]. However, while sparing of the normal hepatic parenchyma adjacent to the tumour is related to the heat-sink effect produced by portal blood flow [25, 27], the limited involvement of normal peritumoural tissue in the lung has been attributed to its high air content, which reduces its electrical conductivity [15]. This hypothesis is consistent with the impedance values recorded in the present study, which are significantly higher than those observed during treatment of liver tumours using the same kind of electrodes and the same RF power [10].

The RF electrodes used were appropriate for the tumours being treated, in that the thermal lesions that could be expected with both types were equal to or larger than the tumours themselves, including the three nodules that displayed incomplete radiological necrosis after the first treatment, all of which measured $3.1-3.5 \mathrm{~cm}$ in diameter. The failure was probably caused by suboptimal placement of the electrode tip within the tumour, which is more frequent when the tumour is irregularly shaped or there are anatomic obstacles (e.g. a rib or shoulder blade) along the electrode-insertion pathway. However, other factors might have been involved, e.g. heat dissipation caused by the presence of large vessels near the tumour or certain characteristics of the tumour tissue itself, such as the presence of necrosis, which is associated with higher-than-expected impedance and premature interruption of RF energy delivery.
The complication rate was very low. Cerebral microembolisation [28], intraparenchymal haemorrhage [29], clinically relevant haemoptysis, intractable cough and dyspnoea [13-17] have been reported in other studies but were not observed in any of the current patients. The most frequent complication was pneumothorax, but its incidence was similar to that observed after lung biopsy [21] and all cases were self-limiting. The local aanesthesia technique used may have contributed to the lower frequency and severity of pneumothorax in the current series compared with those reported by others [13-17]. The local lung compression produced by the xylocaine bolus in the parietal subpleural space reduced the amount of aerated lung parenchyma penetrated by the electrode, thus probably lowering the risk of pneumothorax. General anesthesia is time consuming and increases the complexity and costs of the procedure. In addition, it is unnecessary. RFTA has proved to be virtually painless if performed correctly and an alert patient's report of pain is a highly reliable sign of operator errors. Certain complications reported after RFTA of lung tumours might have been avoided if the patients had been conscious and able to report their sensations during the procedure.

For local control of Stage-I NSCLCs, the results of RFTA are particularly promising. The disease-free survival rate at $1 \mathrm{yr}$ was $60 \%$. Compared with surgery, RFTA is associated with greater sparing of normal parenchyma and less impairment of pulmonary function $[4,7,8]$. RFTA was also highly effective for local control of small pulmonary CRC-METs, which recurred in $<10 \%$ of the cases. The main risk for these patients are new intra- or extrapulmonary metastases, which developed in more than half of the current CRC-METs patients $1 \mathrm{yr}$ after RFTA treatment. As proposed for hepatic CRC-METs [30], a "test-of-time approach" could be adopted for small pulmonary CRC-METs, using RFTA as a less invasive first-line intervention. Thereafter, in the persistent absence of extrapulmonary disease, surgery might be considered for episodes of local recurrence. However, it is important to recall that these events can also be controlled with additional RFTA, which, unlike surgery, can be repeated with a good chance of success, less trauma to the patient and, needless to say, considerably lower costs.

In conclusion, the current authors' experience indicates that radiofrequency thermal ablation can be a safe and effective alternative treatment for the local control of small nonsmall cell lung cancers or pulmonary isolated metastases from colorectal cancer in a highly selected subset of patients without surgical prospects. The clinical impact and long-term results of radiofrequency thermal ablation need to be confirmed in a larger series of patients, and radiofrequency thermal ablation should ideally be compared with surgery.

\section{REFERENCES}

1 Spiro SG, Porter JC. Lung cancer-where are we today? Am J Respir Crit Care Med 2002; 166: 1166-1196.

2 Penna C, Nordlinger B. Colorectal metastasis (liver and lung). Surg Clin North Am 2002; 82: 1075-1090.

3 Ginsberg RJ, Vokes EE, Raben A. Cancer of the lung: non small cell lung cancer. In: Pass HI, Mitchell JB, Johnson DH, Turrisi AT, eds. Lung Cancer: Principles and Practice 
of Oncology. 5th Edn. Philadelphia, Lippincott-Raven, 1996; pp. 849-857.

4 Kearney DJ, Thomas HL, Reilly JJ. Assessment of operative risk in patients undergoing lung resection.Importance of predicted pulmonary function. Chest 1994; 105: 753-759.

5 Vogelsang H, Haas S, Hierholzer C, Berger U, Siewert JR, Prauer H. Factors influencing survival after resection of pulmonary metastases from colorectal cancer. Br J Surg 2004; 91: 1066-1071.

6 Licker M, Spiliopoulos A, Frey JG, et al. Risk factors for early mortality and major complications following pneumonectomy for non-small cell carcinoma of the lung. Chest 2002; 121: 1890-1897.

7 Handy JR, Asaph JW, Skokan L, et al. What happens to patients undergoing lung cancer surgery? Outcomes and quality of life before and after surgery. Chest 2002; 122: 21-30.

8 Spira A, Ettinger DS. Multidisciplinary management of lung cancer. N Engl J Med 2004; 350: 379-392.

9 Rainer F. Stage-III NSCLC: multimodality therapy for inoperable tumors. Lung Cancer 2004; 45: Suppl. 2, 113-123.

10 Rossi S, Buscarini E, Garbagnati F, et al. Percutaneous treatment of small hepatic tumors by an expandable RF needle electrode. AJR Am J Roentgenol 1998; 170: 1015-1022.

11 Curley SA, Izzo F, Delrio P, et al. Radiofrequency ablation of unresectable primary and metastatic hepatic malignancies. Ann Surg 1999; 230: 1-8.

12 Solbiati L, Goldberg SN, Ierace T, et al. Hepatic metastases: percutaneous radio-frequency ablation with cooled-tip electrodes. Radiology 1997; 205: 367-373.

13 Dupuy DE, Zagoria RJ, Akerley W, Mayo-Smith WW, Kavanagh PV, Safran H. Percutaneous radiofrequency ablation of malignancies in the lung. AJR Am J Roentgenol 2000; 174: 57-59.

14 Suh RD, Wallace AB, Sheehan RE, Heinze SB, Goldin JG. Unresectable pulmonary malignancies: CT-guided percutaneous radiofrequency ablation - preliminary results. Radiology 2003; 229: 821-829.

15 Lee JM, Jin GY, Goldberg SN, et al. Percutaneous radiofrequency ablation for inoperable non-small cell lung cancer and metastases: preliminary report. Radiology 2004; 230: 125-134.

16 Gadaleta C, Mattioli V, Colucci G, et al. Radiofrequency ablation of 40 lung neoplasms: preliminary results. AJR Am J Roentgenol 2004; 183: 361-368.

17 Nishida T, Inoue K, Kawata Y, et al. Percutaneous radiofrequency ablation of lung neoplasms: A minimally invasive strategy for inoperable patients. J Am Coll Surg 2002; 195: 426-430.

18 Organ L. Electrophysiologic principles of radiofrequency lesion making. Appl Neurophysiol 1976; 39: 69-76.

19 Miao Y, Ni Y, Bosmans H, et al. Radiofrequency ablation for eradication of pulmonary tumor in rabbits. J Surg Res 2001; 99: 265-271.

20 Marasso A, Bernardi V, Gai R, et al. Radiofrequency resection of bronchial tumors in combination with cryotherapy: evaluation of a new technique. Thorax 1998; 53: 106-109.

21 Gardner D, vanSonnenberg E, D'Agostino HB, Casola G, Taggart S, May S. CT-guided transthoracic needle biopsy. Cardiovasc Intervent Radiol 1991; 14: 17-23.

22 Pauwels RA, Buist AS, Ma P, Jenkins CR, Hurd SS, GOLD Scientific Committee. Global strategy for the diagnosis, management, and prevention of chronic obstructive pulmonary disease: National Heart, Lung, and Blood Institute and World Health Organization Global Initiative for Chronic Obstructive Lung Disease (GOLD): executive summary. Respir Care 2001; 46: 798-825.

23 Mountain CF, Dresler CM. Regional lymph node classification for lung cancer staging. Chest 1997; 111: 1718-1723.

24 Mulier S, Ni Y, Miao Y, et al. Size and geometry of hepatic radiofrequency lesions. Eur J Surg Oncol 2003; 29: 867-878.

25 Rossi S, Garbagnati F, Lencioni R, et al. Percutaneous radiofrequency thermal ablation of nonresectable hepatocellular carcinoma after occlusion of tumor blood flow. Radiology 2000; 217: 119-126.

26 Livraghi T, Golberg SN, Lazzaroni S, Meloni F, Solbiati L, Scott Gazelle G. Small hepatocellular carcinoma: treatment with radiofrequency ablation versus ethanol injection. Radiology 1999; 210: 655-661.

27 Patterson EJ, Scudamore CH, Owen DA, Nagy AG, Buczkowski AK. Radiofrequency ablation of porcine liver in vivo - effects of blood flow and treatment time on lesion size. Ann Surg 1998; 227: 559-565.

28 Rose SC, Fotoohi M, Levin DL, Harrell JH. Cerebral microembolization during radiofrequency ablation of lung malignancies. J Vasc Interv Radiol 2002; 13: 1051-1054.

29 Vaugn C, Mychaskiw G, Sewell P. Massive hemorrhage during radiofrequency ablation of pulmonary neoplasm. Anesth Analg 2002; 94: 1149-1151.

30 Livraghi T, Solbiati L, Meloni F, Ierace T, Goldberg SN, Gazelle GS. Percutaneous radiofrequency ablation of liver metastases in potential candidates for resection: the "testof-time approach". Cancer 2003; 97: 3027-3035. 